\title{
The Grand Challenges of Exoplanets
}

\author{
Steve B. Howell ${ }^{\star t}$ \\ NASA Ames Research Center, Mountain View, CA, United States
}

Keywords: grand challenge, spectroscopy, exoplanets, planets, habitability

\section{INTRODUCTION}

The start of the Exoplanet section of Frontiers in Astronomy and Space Sciences comes at an opportune time in the history of this fledgling field. Starting about 25 years ago, with the discovery of small planets orbiting a pulsar (Wolszczan and Frail, 1992) and soon thereafter the seminal discovery of 51 Peg b (Mayor and Queloz, 1995), exoplanet research today spans many scientific disciplines. The stature of this research area was recently highlighted via the 2019 Nobel prize being awarded to exoplanet researchers Michel Mayor and Didier Queloz ${ }^{1}$.

Exoplanet exploration is truly a world-wide phenomena, a topic of conversation and study in many scholarly areas and at many levels. Science is an obvious area of interest where exoplanets have become the poster child for multi-disciplinary collaborative science, encompassing astronomy, astrobiology, biology, astrophysics, geology, and planetary science to name the major players. But other fields of study, such as philosophy and religion are involved as well. The broad reach and general appeal of exoplanet research stems from a long fascination we have for the night sky. "Are we alone?" that uniquely human question, has deep roots within us all, likely beginning as soon as we looked up at the night sky and wondered.

If we examine the history of major scientific areas of research, those that stemmed from grass root beginnings based on an amazing and unexpected discovery or idea and then quickly proceeded to produce many initial results, we find that such fields of study swiftly gained momentum and became their own research speciality within a few years. From each beginning, scientists migrated to the field, out of curiosity, interest, and desire to make early and major contributions. The rapid re-purposing or development of new tools (e.g., instruments, techniques) quickly led to many new discoveries. Today, scientists are often drawn to a field by the available resources or funding, but as scientists we all love something challenging, a new playground in which to flex our brains and put our experience to work. Taking the scientific topics of relativity, quantum mechanics, and dark energy as examples of new breakthrough areas of science that exploded on the scene, we note that typically about 20-25 years after the stirrings began, major discoveries, deep understanding, and paradigm changing results appeared.

Thus, I fully expect that same revolution to happen in exoplanet research in the next few years. From the NASA Kepler mission (Borucki et al., 2010; Howell et al., 2014), through TESS (Ricker et al., 2016), and CHEOPS (Cessa, 2019), to the next generation of large, specialized instruments and telescopes planned for the ground and space, the field of exoplanet research is moving forward at a fast pace. We have already seen the significant shift from exoplanet discovery to exoplanet characterization.

Exoplanets aims to be a large part of this research endeavor by bringing open access scientific results, covering all aspects of exoplanet and related science to the world. Below, we outline where we are at present and then discuss a number of grand challenges that face the field. These are areas rich in discovery potential and likely to become sub-fields in their own right. So, come along and explore the grand challenges of exoplanet science. Discover for yourself the excitement and opportunities available for scientific study within this research area.

\footnotetext{
${ }^{1}$ For an interesting discussion of the exoplanet discoveries that led up to the Nobel prize, see Josh Winn's editorial-https://
} blogs.scientificamerican.com/observations/who-really-discovered-the-first-exoplanet/. 


\section{WHERE ARE WE NOW?}

Our solar system, with its mostly flat, well-spaced, stable planetary orbits was the end point of theoretical planetary formation and evolution models for many decades. Inner planets being small and rocky and outer planets being massive ice and gas worlds was considered the norm. Well, this view was soon destroyed with the first exoplanet discoveries. From systems containing hot Jupiters, to densely packed resonant systems with many planets orbiting closer to their sun than even Mercury, to super-Earth sized water worlds, the type and variety of exoplanet architectures is highly diverse. Scientifically, I find the breadth of the discovered exoplanet arrangements very exciting. It means we have a lot more to learn and discover-the Universe still holds an abundance of secrets waiting to be found.

Currently, with over 4,000 confirmed exoplanets, the demographics of alien solar systems are beginning to emerge. Typically, the planets orbit in the approximate plane of their sun's equator, planet size generally increases as orbital periods within a system become longer, and small, rocky planets appear to be abundant. There are, however, exceptions to each of these generalizations. To date, no exoplanetary system very similar to our own solar system has been discovered. What does this mean? Is our solar system unique and not the norm after all? Or is it merely that strong observational biases are at play? The latter is the reality as finding a exoplanet system, such as ours is difficult and currently beyond our technical capabilities (Howard et al., 2012). Detection of Earth-size planets in 1-year orbits about a $\mathrm{G}$ star, large gas giants in many decade long outer orbits, and discovering small, low mass planets, such as the Earth orbiting a star, such as our Sun are all challenges we have yet to master. This is especially true if we wish to perform all of these measurements on a single system.

For the exoplanets themselves, variety seems to be the rule. Systems of large gas giants orbiting their host star every few days, planets orbiting perpendicular to their sun's rotation plane, worlds that are disintegrating right before our eyes, or the fact that the most common exoplanet size to be discovered is a type of planet we do not have in our solar system. All of these fundamental properties for the exoplanets and their orbits are waiting to be understood in detail.

But perhaps the largest area of interest today in exoplanet research (as well as within our own solar system), is the search for life ${ }^{2}$. Small Earth and Super-Earth-size rocky planets orbiting within their star's habitable zone are the focus, but exomoons, ocean worlds, and terrestrial planets in very tight orbits around low mass, faint red stars are contenders as well.

Scientists are attacking the search for life in many ways. Astrobiology is looking into the necessary and sufficient conditions that may be needed for life. Biological work on extremophiles that inhabit our Earth are underway as well. Even probing the question of "what is life?" itself has required scientists to reexamine paradigms and doctrines adhered to for centuries. Astrophysicists are approaching the challenge of detecting life on

\footnotetext{
${ }^{2}$ Astrobiology Science Strategy for the Search for Life in the Universe. https://sites. nationalacademies.org/SSB/CurrentProjects/SSB180812.
}

an exoplanet in other ways. One is to build larger telescopes that can collect more light as well as provide higher spatial resolution. Another is building instruments especially designed to measure masses of Earth-size bodies orbiting in the habitable zone of stars like our Sun. This work aims to find the best candidates with the highest potential to be Earth-like. The next generation of extremely large ground-based telescopes (e.g., TMT, E-ELT) now planned are already gearing up to make exoplanet observations a prime research area that they will explore. Large new space telescopes too, such as LUVOIR and HabEx, are being studied and planned for the next decade. Currently, direct images have been obtained for exoplanets orbiting far from their host stars. Using coronagraphic imaging techniques and observing young dust and gas laden stars, such as Fomalhaut and HR 8799, astronomers have obtained optical and IR images showing massive, young (still warm), gas giant planets in distant orbits. While these images are fantastic and provide detailed views of recently formed massive exoplanets, LUVOIR and HabEx have goals of acquiring direct images and atmospheric spectra of the best Earth-like candidate worlds in order to search for the signatures of life.

\section{MOVING FROM DISCOVERY TO CHARACTERIZATION}

The discovery of most exoplanets to date has been accomplished by using the transit technique. By making precise photometric measurements from the ground or in space and waiting and watching to detect the small telltale dip observed in the light curve when an exoplanet crosses its parent star, exoplanets can be found. Transit measurements allow the planet's size, orbital period and semi-major axis to be inferred. The planet size comes directly from the depth of the transit, it being the ratio of the planets area compared to that of is host star. Complications can arise in this simple scheme if the host star is actually a member of a bound or line-of-sight close stellar pair. Thus, knowing the host star properties in detail, e.g., its radius and environment, is a key element in understanding and characterizing any detected exoplanets. Ground-based follow-up observations that provide precise stellar parameters are thus an on-going critical part of exoplanet research (Furlan et al., 2017, 2018).

Radial velocity (RV) discoveries of exoplanets is also a valuable method of discovery. While generally limited, at present, to planets more massive than Earth, this technique provides information that is otherwise unavailable. Doppler measurements can tell us the planets orbit details as well as provide the planets mass (or mass limit). New instrumentation and the next generation of large ground-based telescopes will allow RV discovery of more exoplanets and greatly lower the mass limit currently trending toward Earth mass objects.

Exoplanets discovered by or followed-up with radial velocity measurements which also transit their parent star hold a special place in research. Such planets will have their masses measured (or estimated) and, along with their radius, yield a mean density value allowing further characterization as a rocky planet or an ice or gas giant. The discovery of exoplanets orbiting bright 
host stars has been a boon to finding and characterizing these high-value exoplanets.

Ground- and Space-based microlensing studies provide us a look into more outer solar system regions containing massive planets, somewhat like giving us a view into the nature of our own large gas and ice giants. The NASA WFIRST mission, planned for launch in the mid-2020's, will bring microlensing to bear in a large and systemic way for exoplanet research. We note that the vast majority of currently known exoplanets have orbital semi-major axes values of $<1$ au while microlens detections will generally be at separations of 1 au and larger.

Kepler, TESS, and CHEOPS for example, have/will mostly discover short-period inner planets with their transit observations. Longer period planets, while possibly larger and more massive, are harder to find during short (<years) time visits by current programs. Microlensing detection space, as anticipated with WFIRST, will provide samples of longer period, outer solar system planets (Montet et al., 2017). The connection of these two samples could inform us that large ice or gas giant planets are common orbiting far out from their host star while terrestrial planets orbit closer in. However, the detected microlensed exoplanets will not be members of any radial velocity or transit detected systems, and visa versa, therefore the direct connection of discovered inner and outer planets will not be possible.

A number of single transit events, likely to be due to large, distant exoplanets have been detected by space missions, such as Kepler. Such Single-transit exoplanets imply longer orbital period, outer system planets that could provide a tie to previously detected smaller inner exoplanets within the same system. Even if the single transit cannot provide exquisite details of the orbit, the statistical nature of a set of distant, large planets would be a useful metric to understand outer solar system planets.

Planetary system architectures seem to come in an unimaginable array of types ( $\mathrm{He}$ et al., 2019). Contrary to theoretical expectations, exoplanets have been found in systems thought to be impossible or unlikely. For example, many tightly packed systems have been detected in which mutual gravitational attraction causes measurable orbital effects, such as transit timing variations. Circumbinary planets, such as Kepler 16AB $\mathrm{b}$, a giant planet orbiting far from its close binary host stars, are now not uncommon and being regularly searched for. Exoplanet host stars are known to have a $\sim 46 \%$ binary fraction, leading to binary star systems in which detected planets orbit one of the stars, providing ambiguity in some cases as to which star the detected planet actually orbits. A number of hot Jupiter systems contain a distant stellar companion making us think that perhaps this distant companion is a critical part of the equation. These are just some of the many intriguing questions left to answer about planetary system architectures. Theoretical and observational work are moving from simple discovery to detailed characterization taking on the challenge to answer these questions with research programs focusing on exoplanet formation, dynamics, and evolution.

The synergy between ground and space observations, such as Gaia, Kepler, the VLT, and Gemini observatories can not be ignored if we are to make significant progress of the challenges ahead. Large space missions, such as JWST and big surveys, such as PLATO (Rauer et al., 2016) and WFIRST will provide new and exciting observations adding to our knowledge of exoplanets. But ground-based telescopes will always provide us with more observing time, instrument upgrades on a regular basis including speciality instruments for exoplanet research, novel techniques to remove Earth atmospheric signatures, and larger and larger apertures that increase our light gathering power and overall spatial resolution.

\section{THE SEARCH FOR LIFE}

\subsection{Occurrence Rates}

How many Earth-like exoplanets might exist? This is a fundamental question that drives much of exoplanet research. But first off, what does "Earth-like" mean? A small, rocky planet in the habitable zone of its star? Ok, but does the planet have to orbit a G star? Does the planet need to have plate tectonics, an oxygen rich atmosphere, oceans, land masses, or intelligent life? Some may even think Earth-like means cars and shopping malls. Earth-like can be defined and redefined ad nauseam (see Schwieterman et al., 2019a).

Let us take, for this example, that a starting definition for Earth-like would be a set of exoplanets that are Earth-size and located in or near the habitable zone of their star. We will suspend any details related to the upcoming discussion of possible life in environments, such as Venus or Europa for now. In principle, the occurrence rate of Earth-like planets can be simply determined from the ratio of the number of Earth-size planets we detect with orbits that place them in the habitable zone of their host star compared with the number of stars we have observed for which a Earth-size planet transit could have been detected. Well, once you begin to look in detail at these simple quantities you find they are not so simple. We do not have perfect knowledge of either part of this ratio due to completeness and reliability, that is, what exoplanets did we miss and is every detected signal a real exoplanet.

This is a highly active and debated area of current exoplanet research and numerous publications address the details and formulate answers. Taking the overall best current estimates for Earth-like exoplanets, we find an occurrence rate of about 0.150.35 Earth-like planets orbiting every G star and about 0.25 0.50 Earth-like planets orbiting every $\mathrm{M}$ star. Broadly taking these numbers as $\sim 50 \%$ and the fact that there are $\sim 250$ billion stars in the Milky Way, these numbers mean that there are $\sim 125$ billion Earth-like planets in our Galaxy. While this sounds like a lot of Earth-like planets and one might take it as ample evidence that life exists elsewhere, our challenge as an exoplanet scientist is to identify the true Earth-like exoplanet candidates we can observe in detail (far less than 125 billion) and work toward technologies and observations of such exoplanets that will provide unambiguous detection of alien life.

\subsection{Habitable Environments}

What makes a planet habitable? This question has gone from the simplistic view of having liquid water on its surface to a 
TABLE 1 | A selection of top-level exoplanet challenges.

\begin{tabular}{lll}
\hline Exomoons & Future ground-based exoplanet instrumentation & Synergy of transits and RVs \\
Disintegrating exoplanets & M stars and habitability & Know thy star, know thy planet \\
Hot and warm exo-jupiters & The promise of microlensing & Exoplanets in binary star systems \\
Exoplanets in star clusters & Exoplanets and religion/philosophy & Exoplanetary system architectures \\
Circumbinary exoplanets & Exoplanets and the Drake equation & Exoplanet formation and dynamics \\
Ultra-short period exoplanets & Atmospheric characterization & Metallicities of exoplanet hosts \\
Statistical methods in exoplanets & Asteroseismology of exoplanet host stars & Exoplanet habitability \\
Venus as an exoplanet & Multi-wavelength views of exoplanets & The promise of space telescopes
\end{tabular}

vast array of connected conditions which are thought to be required. Additional confusion and complexity in this topic can arise when one incorporates non-human-like life, such as tube worms living in deep sea vents, bacteria discovered in the mud at the bottom of the Marianas trench, the possibility of ocean life under the ice on the moon Europa or bacteria floating in the Venusian atmosphere.

Can life exist on a rocky, habitable zone world orbiting a flaring $\mathrm{M}$ star? What time period is required for life to form and evolve? The ancient Earth was clearly not a place we would enjoy today, so long-term planetary environmental changes are important to understand as well (Schwieterman et al., $2019 b$ ). Such questions as these illustrate the strong relation of biology, astrobiology, and planetary science to exoplanet research. These connections across a multitude of scientists and scientific disciplines makes exoplanet research both world-wide and highly diverse.

\subsection{Atmospheric Spectroscopy of Exoplanets}

There are many reasons why measuring the atmospheric composition of an exoplanet is important. One that stands out in my mind is illustrated within our own solar system, that is, the perplexing situation of Earth and Venus. Here we have two worlds both similar in size and mass, both in/near the habitable zone, both looking to be potentially Earth-like exoplanets if observed from afar, yet so extremely different in their atmospheric composition and surface environment at the present epoch. What happened to Venus? Or is it what happened to the Earth to lead to such differences? How can we use this local example and apply that knowledge to exoplanet study? The study of Venus and its evolution has taken on renewed interest in recent years especially when one thinks about Venus as exoplanet (Kane et al., 2019).

Current exoplanet atmospheric work mainly focuses on ice and giant planets, those for which optical and IR spectra during transit or in reflected light can be obtained and can reveal their atmospheric signatures (see Gauza et al., 2015). Clouds or no clouds, water vapor, and other molecular species have been detected so far, many by using the Hubble Space telescope and a variety of large ground-based telescopes. Going forward, new instrumentation and larger telescopes on the ground offer the promise of obtaining atmospheric signatures for smaller exoplanets, perhaps even super-Earths. IR spectroscopy will be increasingly important due to the atmospheric temperatures involved.

Missions, such as K2, TESS, and CHEOPS are currently providing the best exoplanet targets for atmospheric study to date, those with bright, nearby host stars. Future space missions, such as Ariel (scheduled to launch in 2028) and beyond, are planning to obtain detailed atmospheric spectra of hundreds of alien worlds in order to characterize the planets and continue the search for life. Direct images of small planets orbiting sun-like stars are planned as well using space telescopes set to launch in the 2030's.

In addition to the observational work to measure an exoplanet atmosphere, laboratory astrophysics that will produce spectral libraries of relevant species and theoretical calculations providing line lists and cross sections are greatly needed as well. Exoplanet atmospheres, especially those that are hot or warm, are a complex mixture of non-equilibrium molecular chemistry. Model calculation and interpretation of atmospheric observations must take into account the scale height as well as the radiative and dynamic timescales involved. The ratio, $\tau_{\text {radiative }} / \tau_{\text {dynamic }}$, is proportional to $g$, the acceleration of gravity, which in turn depends on $\mathrm{M}_{\text {planet }} / \mathrm{R}_{\text {planet }}^{2}$, pointing back again to the need for highly accurate measurements of the host star properties (e.g., radius) and the planets mass.

\section{SUMMARY}

The brief synopsis above has discussed many of the grand challenges we face together in exoplanet research. Such topics are ripe to become exciting areas of focused research in the field. Table 1 provides a topical list of some relevant top exoplanet challenges that face us today.

NASA and ESA's space exoplanet research programs, consisting of Hubble, Spitzer, CoRoT, Kepler/K2, Gaia, TESS, JWST, WFIRST, CHEOPS, PLATO, ARIEL, and future proposed concepts, such as New Worlds Telescope, and LUVOIR, along with current and planned large ground-based telescopes around the world, will provide new and exciting exoplanet research opportunities for decades to come. New instrumentation recently put in place and or being developed is a valuable asset as well. However, the planning for and making use of ground-based assets in relation to space missions is often fragmentary due 
to proposal cycles and funding mechanisms. As a community, we should work to bring these resources together in order to perform coordinated and detailed exoplanet studies.

The famous Drake equation tries to estimate the number of active, communicative alien civilizations within the Milky Way. But what it does not address is the vast distances which lie between the stars. These regions are far emptier, in a relative sense, than even the empty space in atoms. Thus, it is likely that the Earth will be the only home we ever have and that remote sensing will be our sole opportunity to study the distant worlds. I am hopeful that our mutual world-wide study of exoplanets, within these open access scientific pages, will allow us to realize how lucky and special we are here on Earth.

There are other challenges as well in addition to scientific exoplanet research. We have the challenge to be inclusive of world-wide research and bring it into our peer-reviewed literature, we have the challenge of a multigenerational workforce, and we all have the challenge

\section{REFERENCES}

Borucki, W. J., Koch, D., Basri, G., Batalha, N., Brown, T., Caldwell, D., et al. (2010). Kepler planet-detection mission: introduction and first results. Science 327:977. doi: 10.1126/science. 1185402

Cessa, V. (2019). CHEOPS mission overview. Proc. SPIE 11180:111803L. doi: $10.1117 / 12.2536048$

Furlan, E., Ciardi, D. R., Cochran, W. D., Everett, M. E., Latham, D. W., Marcy, G. W., et al. (2018). The Kepler follow-up observation program. II. Stellar parameters from medium- and high-resolution spectroscopy. Astrophys. J. 861:22. doi: 10.3847/1538-4357/aaca34

Furlan, E., Ciardi, D. R., Everett, M. E., Saylors, M., Teske, J. K., Horch, E. P., et al. (2017). The Kepler follow-up observation program. I. A catalog of companions to Kepler stars from high-resolution imaging. Astron. J. 153:28. doi: 10.3847/1538-3881/153/2/71

Gauza, B., Béjar, V. J. S., Pérez-Garrido, A., Osorio, M. R. Z., Lodieu, N., Rebolo, R., et al. (2015). Discovery of a young planetary mass companion to the nearby M Dwarf VHS J125601.92-125723.9. Astrophys. J. 804:96. doi: 10.1088/0004-637X/804/2/96

He, M. Y., Ford, E. B., and Ragozzine, D. (2019). Architectures of exoplanetary systems-I. A clustered forward model for exoplanetary systems around Kepler's FGK stars. Monthly Notices R. Astron. Soc. 490, 4575-4605. doi: $10.1093 / \mathrm{mnras} / \mathrm{stz} 2869$

Howard, A. W., Marcy, G. W., Bryson, S. T., Jenkins, J. M., Rowe, J. F., Batalha, N. M., et al. (2012). Planet occurrence within $0.25 \mathrm{AU}$ of solar-type stars from Kepler. Astrophys. J. Suppl. 201:20. doi: 10.1088/0067-0049/201/2/15

Howell, S. B., Sobeck, C., Haas, M., Still, M., Barclay, T., Mullally, F., et al. (2014). The K2 mission: characterization and early results. Publ. Astron. Soc. Pac. 126:398. doi: 10.1086/676406

Kane, S. R., Arney, G., Crisp, D., Domagal-Goldman, S., Glaze, L. S., Goldblatt, C., et al. (2019). Venus as a laboratory for exoplanetary of bias. However, we have the great benefit of diverse, active, and collaborative scientists from around the world and I look forward to working together toward a better future in which we have access to and cherish each others exoplanet research.

\section{AUTHOR CONTRIBUTIONS}

The author confirms being the sole contributor of this work and has approved it for publication.

\section{ACKNOWLEDGMENTS}

I would like to thank Claudio Bogazzi and Rebecca Smith for their support in starting this new Frontiers section on Exoplanets and for the generous amount of time they gave to me. I also wish to acknowledge the word-wide exoplanet community for their contributions to research in the field of exoplanets.

science. J. Geophys. Res. Planets 124, 2015-2028. doi: 10.1029/2019JE0 05939

Mayor, M., and Queloz, D. (1995). A jupiter-mass companion to a solar-type star. Nature 378, 355-359. doi: 10.1038/378355a0

Montet, B. T., Yee, J. C., and Penny, M. T. (2017). Measuring the galactic distribution of transiting planets with WFIRST. Publ. Astron. Soc. Pac. 129:044401. doi: 10.1088/1538-3873/aa57fb

Rauer, H., Aerts, C., Cabrera, J., and The PLATO Team (2016). The PLATO mission. Astron. Nachr. 337:961. doi: 10.1002/asna.201612408

Ricker, G. R., Vanderspek, R., Winn, J., Seager, S., Berta-Thompson, Z., Levine, A., et al. (2016). The transiting exoplanet survey satellite. Proc. SPIE 9904:99042B. doi: $10.1117 / 12.2232071$

Schwieterman, E. W., Reinhard, C. T., Olson, S. L., Harman, C. E., and Lyons, T. W. (2019b). A limited habitable zone for complex life. Astrophys. J. 878:19. doi: 10.3847/1538-4357/ab1d52

Schwieterman, E. W., Reinhard, C. T., Olson, S. L., Ozaki, K., Harman, C. E., Hong, P. K., et al. (2019a). Rethinking CO antibiosignatures in the search for life beyond the solar system. Astrophys. J. 874:9. doi: 10.3847/1538-4357/ab05e1

Wolszczan, A., and Frail, D. (1992). A planetary system around the millisecond pulsar PSR1257 + 12. Nature 355, 145-147. doi: 10.1038/355145a0

Conflict of Interest: The author declares that the research was conducted in the absence of any commercial or financial relationships that could be construed as a potential conflict of interest.

Copyright (C) 2020 Howell. This is an open-access article distributed under the terms of the Creative Commons Attribution License (CC BY). The use, distribution or reproduction in other forums is permitted, provided the original author(s) and the copyright owner(s) are credited and that the original publication in this journal is cited, in accordance with accepted academic practice. No use, distribution or reproduction is permitted which does not comply with these terms. 